\title{
Prognostic Factors and Predictors of In-Hospital Mortality Among COVID-19 Patients Admitted to the Intensive Care Unit: An Aid for Triage, Counseling, and Resource Allocation
}

Waleed Burhamah ${ }^{1}$, Iman Oahi ${ }^{2}$, Melinda Oroszlányová ${ }^{3}$, Sameera Shuaibi ${ }^{4}$, Razan Alhunaidi ${ }^{5}$, May Alduwailah $^{5}$, Maryam Alhenaidi ${ }^{5}$, Zahraa Mohammad ${ }^{5}$

1. School of Medicine, Royal College of Surgeons in Ireland, Dublin, IRL 2. Department of General Surgery, Mubarak AlKabeer Hospital, Kuwait, KWT 3. College of Engineering and Technology, American University of the Middle East, Kuwait, KWT 4. Department of Internal Medicine, Al-Adan Hospital, Kuwait, KWT 5. Department of Internal Medicine, Kuwait University, Health Sciences Center, School of Medicine, Kuwait, KWT

Corresponding author: Waleed Burhamah, waleedburhamah1994@gmail.com

\section{Abstract}

Background: The severe acute respiratory syndrome coronavirus 2 (SARS-CoV-2) remains today a global health pandemic. Those with severe infection are at risk of rapid clinical deterioration; as a result, intensive care unit (ICU) admission is not uncommon in such patients. A number of determinants have been identified as predictors of poor prognosis and in-hospital mortality, ranging from demographic characteristics, laboratory and/or radiological findings.

Aim: To identify determinants of in-hospital mortality and examine the accuracy of seven early warning scores in predicting in-hospital mortality.

Methods: This is a retrospective study conducted in Kuwait from July 2020 to March 2021, and participants were adult patients with a positive test on the real-time polymerase chain reaction (RT-PCR) for SARS-CoV-2 and who met the criteria for ICU admission. Data collected included: demographics, clinical status on hospital arrival, laboratory test results, and ICU course. Furthermore, we calculated seven early warning scores for each of our patients.

Results: A total of 133 patients were admitted to our COVID-19 ICU with a median age of 59 years. Arrival to ICU on mechanical ventilation (MV), developing in-hospital complications, having chronic kidney disease (CKD), having a high white blood count (WBC), lactate dehydrogenase (LDH), lactate, or urea levels were found to be significant predictors of in-hospital mortality. Furthermore, the 4C mortality score for COVID19, VACO index for COVID-19 mortality, and the PRIEST COVID-19 clinical severity score proved to be the most superior in predicting in-hospital mortality.

Review began 07/05/2021 Review ended 07/12/2021 Published 07/23/2021

\section{๑) Copyright 2021}

Burhamah et al. This is an open access article distributed under the terms of the Creative Commons Attribution License CC-BY 4.0., which permits unrestricted use, distribution, and reproduction in any medium, provided the original author and source are credited.
Conclusion: Identifying high-risk patients and those with a poor prognosis allows for efficient triaging and the delivery of high-standard care while minimizing the strain on the healthcare system.

Categories: Anesthesiology, Emergency Medicine, Internal Medicine

Keywords: covid-19, icu, critical care, sars-cov- 2, prognostic scores

\section{Introduction}

In December 2019, a cluster of cases of pneumonia of unknown etiology was reported in the City of Wuhan, China. Shortly, the novel severe acute respiratory syndrome coronavirus 2 (SARS-CoV- 2) was identified as the culprit. It remains today a global health pandemic responsible for more than 3 million deaths worldwide [1].

This virus is a newly identified strain that belongs to a family of viruses known as the Coronaviruses. Seven are known to cause disease in humans, including the culprits behind the outbreaks of severe acute respiratory syndrome (SARS) and middle east respiratory syndrome (MERS) [2]. The mode of human-tohuman transmission is mainly through respiratory secretions [3]. Various incubation periods have been reported by numerous studies, a period of 2 to 14 days seems to be the general consensus [4,5]. Clinically, it manifests as a syndrome with diverse symptomatology and a spectrum of severities, ranging from being asymptomatic or merely a flu-like illness to as devastatingly as respiratory failure, septic shock, multiple organ dysfunction, and death [6,7]. Of the COVID-19 patients requiring hospital admission, it is estimated that $25 \%$ also meet the requirements for intensive care unit (ICU) admission [8,9].

A number of determinants have been identified as indicators of severe infection and poor prognosis. 
Patients with severe COVID-19 infection are a group of high-risk patients, they initially manifest with a clinical picture of high respiratory rate (usually $\geqslant 30$ breaths per minute), oxygen saturation of $\leqslant 93 \%$ and lung infiltrates $>50 \%$ on chest X-ray [10]. Other indicators of a severe infection include high fever $\left(>39^{\circ} \mathrm{C}\right)$, lymphopenia, elevated liver and renal function tests (aspartate aminotransferase, alanine aminotransferase, creatinine, and urea), elevated acute-phase reactants (C-reactive protein (CRP), procalcitonin (PCT), and serum ferritin), and elevated coagulation profile parameters (prothrombin time, fibrinogen, and D-dimer) [11]. This subset of patients are at high risk for rapid clinical deterioration and the development of respiratory failure [9] Furthermore, a number of early warning scores have been generated and validated for use in COVID-19 patients [12-18]; they each differ in clinical variables but ultimately aid in the prediction of patient outcomes and in-hospital mortality.

During a pandemic involving a novel pathogen with unknown virulence and that presents with a spectrum of severities, a strain on the healthcare system and resources available is inevitable. As a result, risk stratification and efficient triaging are crucial; this allows adequate resource allocation in order to deliver a high standard of care that is patient-centered. Furthermore, it aids in the counseling of the patient's relatives to relay a more realistic picture regarding the outcomes of the patient.

On February 24, 2019, the Kuwait News Agency (KUNA) reported the first few cases of the SARS-CoV- 2 virus in Kuwait, identified in a number of travelers arriving from abroad. Despite the efforts to limit the spread of this virus, the number of cases escalated and as of today has amounted to 294,694 cases and 1703 mortalities [19]. This is a single-center study conducted in Kuwait. In this study, we aim to describe the characteristics of the patients admitted to our COVID-19 intensive care unit (ICU). Second, we aim to identify the determinants of in-hospital mortality. Lastly, we examine and compare the accuracy of a number of early warning scores, investigating their performance in our cohort.

\section{Materials And Methods Study design and participants}

This is a retrospective observational study conducted at a university-affiliated hospital in Kuwait, during the period of July 2020 to March 2021. At our hospital, we allocated two of our internal medicine wards and one of our ICUs to the care of COVID-19 patients. Our dedicated COVID-19 ICU is composed of 25 beds and is run by a team of certified ICU staff, internal medicine residents, and nursing staff that provide one-to-one patient care around the clock. Study participants were adult patients (above 18 years of age) with a positive test on the real-time polymerase chain reaction (RT-PCR) for the SARS-CoV-2 virus, and who met the criteria for ICU admission either from the emergency department (ED) or the COVID-19 wards. Ethical approval was obtained from the ethical committee in the Ministry of Health, Kuwait.

\section{Data collection}

We reviewed the clinical records of our ICU patients. The following data were collected: demographics (age, gender, and nationality), comorbidities, smoking history, and the use of angiotensin-converting enzyme inhibitors (ACEI). We recorded the patient's vital signs and symptoms upon hospital arrival as well as the duration of symptoms (days). Furthermore, details regarding the ICU course were recorded including, the source of ICU admission (ward vs. ED), the mode of ventilation on ICU arrival (MV vs. NIV), laboratory test results on day 1 of ICU, need for mechanical ventilation (MV) during ICU stay, duration of MV (days), length of ICU stay (days), and treatments received. Our primary outcome was in-hospital mortality.

\section{Early warning scores}

For each of our patients, we calculated seven early warning scores that have been previously validated. The Modified Early Warning Score (MEWS) for Clinical Deterioration [12], the National Early Warning Score 2 (NEWS2) [13], the Quick COVID-19 Severity Index (qCSI) [14], the International Severe Acute Respiratory Infection Consortium Clinical Characterization Protocol (4C mortality score for COVID-19) [15], the Veterans Health Administration COVID-19 (VACO) Index for COVID-19 Mortality [16], The MuLBSTA Score for Viral Pneumonia Mortality (MulBTSA) [17], and the Pandemic Respiratory Infection Emergency System Triage Severity Score (PRIEST) [18].

\section{Statistical analysis}

The analysis of our data was performed using R software, version 4.0.5 (R Foundation for Statistical Computing, Vienna, Austria). Continuous variables were reported as median (interquartile range - IQR), and categorical variables were reported as frequency (percentage). In order to compare the clinical records of patients who were discharged and those who died, the continuous variables were tested by the t-test and Mann-Whitney test (at $\alpha=0.05$ ), and the categorical variables were tested by using the Chi-squared test (at $\alpha=0.05$ ). To determine potential risk factors for in-hospital death, the odds ratios (OR) were calculated with the $95 \%$ confidence intervals (CI) and the corresponding p-values. Furthermore, the Area Under the Receiver Operating Characteristics (AUROC) metric was calculated for each mortality score to evaluate its performance in predicting in-hospital mortality. Youden's index was used to estimate optimal cut-off points and corresponding sensitivity and specificity. 


\section{Cureus}

\section{Results}

A total of 133 patients were admitted to our COVID-19 ICU with a median age of 59 years and a median length of stay of seven days. The majority (65\%) were transferred from the COVID-19 wards after deteriorating and only $35 \%$ were directly admitted to ICU from the ED. Most of the patients were males (68\%), non-Kuwaiti (68\%), and non-smokers (90\%). Less than half of the patients (41\%) survived, and 59\% died in the hospital (Table 1). The majority of our cohort had medical comorbidities (84\%), with the most common being diabetes mellitus (57\%) followed by hypertension (55\%; Table 2). In addition, the most common symptoms on hospital admission were shortness of breath (73\%), fever (68\%), and cough (60\%), with a median duration of four days (Table 3). Vital signs on hospital arrival are summarized in Table 4 and the day 1 laboratory results are summarized in Table 5 .

$N=133^{7}$

\section{Nationality}

Kuwaiti

Non-Kuwaiti

$91(68 \%)$

Age

$59(49,68)$

Gender

Female

Male

$90(68 \%)$

Smoker

No

$120(90 \%)$

Yes

Direct admission to ICU

No (ward first)

Yes (from ED to ICU)

Length of ICU stay (days)

$7(3,13)$

Outcome

Death

Discharged

\section{TABLE 1: Clinical characteristics of the patients}

$7 \mathrm{n}(\%)$; median (IQR). 


\section{Cureus}

Comorbidities

$N=133$

Previously healthy

No

Yes

$112(84 \%)$

$21(16 \%)$

Diabetes mellitus

Yes

Hypertension

Yes

$73(55 \%)$

Taking an ACEI

Yes

Dyslipidemia

Yes

Ischemic heart disease

Yes

Asthma

Yes

COPD

Yes

CKD

Yes

Malignancy

Yes

$5(3.8 \%)$

OSA

Yes

$13(9.8 \%)$

Rheumatologic condition

Yes

$3(2.3 \%)$

Hypothyroidism

Yes

$12(9.0 \%)$

\section{TABLE 2: Co-morbidities}

7 n (\%); median (IQR).

ACEI: angiotensin-converting enzyme inhibitor, COPD: chronic obstructive pulmonary disease, CKD: chronic kidney disease, OSA: obstructive sleep apnea. 


\section{Cureus}

\begin{tabular}{|l|l}
\hline Symptoms on hospital admission & $\mathbf{N}=\mathbf{1} 33^{\mathbf{7}}$ \\
\hline Duration of symptoms (days) & $4.0(1.0,6.0)$ \\
Cough & $80(60 \%)$ \\
Fever & $90(68 \%)$ \\
Shortness of breath & $97(73 \%)$ \\
Headache & $3(2.3 \%)$ \\
Chest pain & $8(6.0 \%)$ \\
Arthralgia/myalgia & $6(4.5 \%)$ \\
Vomiting & $16(12 \%)$ \\
Diarrhea & $13(9.8 \%)$ \\
Fatigue & $31(23 \%)$ \\
Altered consciousness & $12(9.0 \%)$
\end{tabular}

\section{TABLE 3: Symptoms on hospital admission}

${ }^{7} \mathrm{n}$ (\%); median (IQR).

$\begin{array}{ll}\text { Vitals on hospital arrival } & \mathbf{N}=133^{7} \\ \text { HR } & 102(89,119) \\ \text { Unknown } & 11 \\ \text { SBP } & 128(116,148) \\ \text { Unknown } & 10 \\ \text { DBP } & 74(66,82) \\ \text { Unknown } & 10 \\ \text { Oxygen Sat on RA } & 88(82,90) \\ \text { Unknown } & 12 \\ \text { Temperature } & 37.90(37.20,38.70) \\ \text { Unknown } & 14 \\ \text { RR } & 30(22,38) \\ \text { Unknown } & 55\end{array}$

\section{TABLE 4: Vital signs on hospital arrival}

7n (\%); median (IQR).

HR: heart rate, SBP: systolic blood pressure, DBP: diastolic blood pressure, Sat on RA: saturation on room air, RR: diastolic blood pressure. 


\section{Cureus}

\begin{tabular}{|c|c|}
\hline Investigations & $N=133^{7}$ \\
\hline $\mathrm{Hb}(\mathrm{g} / \mathrm{L})$ & $126(108,138)$ \\
\hline WBC $\left(10^{9} / \mathrm{L}\right)$ & $11(8,16)$ \\
\hline Lymphocyte count $\left(10^{9} / \mathrm{L}\right)$ & $0.80(0.50,1.40)$ \\
\hline Platelet count $\left(10^{9} / \mathrm{L}\right)$ & $250(179,345)$ \\
\hline CRP (mg/L) & $118(70,211)$ \\
\hline Unknown & 17 \\
\hline PCT (ng/mL) & $1(0,2)$ \\
\hline Unknown & 12 \\
\hline D-dimer (ng/mL) & $791(447,3,070)$ \\
\hline Unknown & 13 \\
\hline LDH (IU/L) & $622(432,832)$ \\
\hline Unknown & 23 \\
\hline Ferritin (ng/L) & $773(382,1,500)$ \\
\hline Unknown & 26 \\
\hline Lactate (mmol/L) & $2.12(1.37,3.05)$ \\
\hline Unknown & 33 \\
\hline Creatinine $(\mu \mathrm{mol} / \mathrm{L})$ & $89(72,136)$ \\
\hline Urea (mmol/L) & $12(8,18)$ \\
\hline ALT (IU/L) & $40(26,72)$ \\
\hline AST (IU/L) & $58(37,88)$ \\
\hline Albumin (g/L) & $31.0(26.0,34.0)$ \\
\hline Troponin (ng/L) & $35(13,190)$ \\
\hline Unknown & 6 \\
\hline
\end{tabular}

\section{TABLE 5: Laboratory results on day 1}

7n (\%); median (IQR).

Hb: hemoglobin, WBC: white blood count, CRP: C-reactive protein, PCT: procalcitonin, LDH: lactate dehydrogenase, ALT: alanine transaminase, AST: aspartate aminotransferase.

Upon arrival to the ICU, $47 \%$ of the patients were already on MV but eventually, $74 \%$ of the ICU patients required MV (Table 6). In terms of management, most of the patients received antibiotics (95\%), 20\% received Oseltamivir, and only $5.3 \%$ received both Oseltamivir and Kaletra. Additionally, half of our patients received Dexamethasone (50\%; Table 7). The majority of our cohort developed in-hospital complications (78\%), with the most common being acute kidney injury (AKI; 46\%), coagulopathy (14\%), and upper gastrointestinal bleeding (8.3\%). Almost half of the patients developed septic shock (47\%; Table 8 ). 


\section{Cureus}

Ventilation

Arrival at ICU on

MV

$62(47 \%)$

NIV

$71(53 \%)$

Eventually required MV

No

$35(26 \%)$

Yes

$98(74 \%)$

Intubated on ICU on day

$1.00(1-3)$

Duration of MV (days)

5.00(3-10.75)

Tracheostomy

No

128(96\%)

Yes

$5(3.8 \%)$

Extubated successfully

No

$75(56 \%)$

Was never intubated

$35(26 \%)$

Yes

$23(17 \%$

\section{TABLE 6: Ventilation}

MV: mechanical ventilation, NIV: non-invasive ventilation. 


\section{Cureus}

Treatments

$N=133^{7}$

Antibiotics

No

Yes

Antiviral

No

Oseltamivir

Oseltamivir and Lopinavir/Ritonavir

Plasma exchange

No

Yes

Tocilizumab

No

Yes

Dexamethasone

No

Yes

Anakinra

No

Yes

IVIG

No

Yes

NO

No

Yes

Proning

No

Yes
$114(86 \%)$

$19(14 \%)$

$6(4.5 \%)$

127 (95\%)

$100(75 \%)$

26 (20\%)

7 (5.3\%)

$113(85 \%)$

20 (15\%)

$67(50 \%)$

$66(50 \%)$

127 (95\%)

$6(4.5 \%)$

130 (98\%)

$3(2.3 \%)$

115 (86\%)

18 (14\%)

$80(60 \%)$

53 (40\%)

\section{TABLE 7: Treatments}

${ }^{7} \mathrm{n}(\%)$; median (IQR).

IVIG: intravenous immunoglobulin, NO: nitric oxide. 


\section{Cureus}

\section{Complications}

In-hospital complications

No

Yes

In-hospital AKI

In-hospital cardiac arrest with ROSC

$10(7.5 \%)$

In-hospital coagulopathy

Types of coagulopathies

DIC

DIC and LL ischemia

DIC, LL ischemia, bowel ischemia, and renal ischemia

DVT

LL ischemia

No

PE

In-hospital MI

$8(6.0 \%)$

In-hospital CVA

In-hospital pneumothorax

$8(6.0 \%)$

In-hospital UGI

In-hospital rhabdomyolysis

In-hospital DKA

$4(3.0 \%)$

In-hospital infective endocarditis

Shock

Cardiogenic shock

No

$66(50 \%)$

Septic shock

$62(47 \%)$

\section{TABLE 8: Complications}

7n (\%); median (IQR).

AKI: acute kidney injury, ROSC: return of spontaneous circulation, DIC: disseminated intravascular coagulation, LL: lower limb, DVT: deep vein thrombosis, PE: pulmonary embolism, MI: myocardial infarction, CVA: cerebrovascular accident, UGI: upper gastrointestinal bleeding, DKA: diabetic ketoacidosis.

Table 9 shows the comparison in the clinical characteristics between the two groups of patients; those who died in the hospital and those who were discharged. The average age was almost ten years higher for the patients who died (62.6 years) and the majority of deaths were males. The symptoms and their average duration were similar. Most of the vital signs on hospital arrival were on average similar between the two groups. The average white blood cell count (WBC), lactate dehydrogenase (LDH), lactate, and urea levels were significantly higher in patients who died than those who were discharged.

\section{Characteristics}

Nationality

\section{Death $(\mathrm{N}=78)$}

Discharged $(\mathrm{N}=55)$

p-Value

0.236 


\section{Cureus}

\begin{tabular}{|c|c|c|c|}
\hline Kuwaiti & $21(26.9 \%)$ & $21(38.2 \%)$ & \\
\hline Non-Kuwaiti & 57 (73.1\%) & 34 (61.8\%) & \\
\hline Age $^{\star}$ & $62.6(13.7)$ & $53.2(14.5)$ & $<0.001$ \\
\hline Gender & & & 0.787 \\
\hline Female & $24(30.8 \%)$ & $19(34.5 \%)$ & \\
\hline Male & $54(69.2 \%)$ & $36(65.5 \%)$ & \\
\hline Smoker & & & 0.505 \\
\hline No & $72(92.3 \%)$ & 48 (87.3\%) & \\
\hline Yes & $6(7.69 \%)$ & $7(12.7 \%)$ & \\
\hline Direct admission to ICU & & & 0.016 \\
\hline No (ward first) & $58(74.4 \%)$ & $29(52.7 \%)$ & \\
\hline Yes (from ED to ICU) & $20(25.6 \%)$ & $26(47.3 \%)$ & \\
\hline Length of ICU stay (days) & $8.83(7.29)$ & $10.7(10.1)$ & 0.239 \\
\hline Previously healthy & & & 0.567 \\
\hline No & $64(82.1 \%)$ & $48(87.3 \%)$ & \\
\hline Yes & 14 (17.9\%) & 7 (12.7\%) & \\
\hline Comorbidities & Death $(\mathrm{N}=78)$ & Discharged (N=55) & $\mathrm{p}$-Value \\
\hline DM & & & 0.741 \\
\hline No & $32(41.0 \%)$ & $25(45.5 \%)$ & \\
\hline Yes & $46(59.0 \%)$ & 30 (54.5\%) & \\
\hline Hypertension & & & 0.55 \\
\hline No & $33(42.3 \%)$ & $27(49.1 \%)$ & \\
\hline Yes & $45(57.7 \%)$ & $28(50.9 \%)$ & \\
\hline Taking ACEI & & & 0.026 \\
\hline No & $63(80.8 \%)$ & $34(61.8 \%)$ & \\
\hline Yes & $15(19.2 \%)$ & $21(38.2 \%)$ & \\
\hline Dyslipidemia & & & 0.703 \\
\hline No & 59 (75.6\%) & $44(80.0 \%)$ & \\
\hline Yes & 19 (24.4\%) & $11(20.0 \%)$ & \\
\hline Ischemic heart disease & & & 0.167 \\
\hline No & $56(71.8 \%)$ & $46(83.6 \%)$ & \\
\hline Yes & $22(28.2 \%)$ & $9(16.4 \%)$ & \\
\hline Asthma & & & 0.266 \\
\hline No & $68(87.2 \%)$ & $52(94.5 \%)$ & \\
\hline Yes & $10(12.8 \%)$ & 3 (5.45\%) & \\
\hline COPD & & & 0.642 \\
\hline No & 75 (96.2\%) & $54(98.2 \%)$ & \\
\hline Yes & 3 (3.85\%) & $1(1.82 \%)$ & \\
\hline CKD & & & 0.02 \\
\hline No & $59(75.6 \%)$ & $51(92.7 \%)$ & \\
\hline
\end{tabular}




\section{Cureus}

Yes

Malignancy

No

Yes

OSA

No

Yes

Rheumatologic condition

No

Yes

Hypothyroidism

No

Yes

Symptoms on hospital admission

Duration of symptoms (days)*

Cough

No

Yes

Fever

No

Yes

Shortness of breath

No

Yes

Headache

No

Yes

Chest pain

No

Yes

Arthralgia/myalgia

No

Yes

Vomiting

No

Yes

Diarrhea

No

Yes
$19(24.4 \%) \quad 4(7.27 \%)$

$75(96.2 \%) \quad 53(96.4 \%)$

$3(3.85 \%) \quad 2(3.64 \%)$

1

$70(89.7 \%) \quad 50(90.9 \%)$

$8(10.3 \%) \quad 5(9.09 \%)$

$75(96.2 \%) \quad 55(100 \%)$

$3(3.85 \%)$

$0(0.00 \%)$

0.552

$72(92.3 \%) \quad 49(89.1 \%)$

$6(7.69 \%) \quad 6(10.9 \%)$

Death $(\mathrm{N}=78) \quad$ Discharged $(\mathrm{N}=55) \quad \mathrm{p}$-Value

$\begin{array}{lll}4.69(4.69) & 3.98(3.21) & 0.302\end{array}$

0.569

$29(37.2 \%) \quad 24(43.6 \%)$

$49(62.8 \%) \quad 31(56.4 \%)$

0.915

$26(33.3 \%) \quad 17(30.9 \%)$

$52(66.7 \%) \quad 38(69.1 \%)$

$21(26.9 \%) \quad 15(27.3 \%)$

$57(73.1 \%) \quad 40(72.7 \%)$

$77(98.7 \%) \quad 53(96.4 \%)$

$1(1.28 \%) \quad 2(3.64 \%)$

0.065

$\begin{array}{ll}76(97.4 \%) & 49(89.1 \%) \\ 2(2.56 \%) & 6(10.9 \%)\end{array}$

$74(94.9 \%) \quad 53(96.4 \%)$

$4(5.13 \%) \quad 2(3.64 \%)$

$68(87.2 \%) \quad 49(89.1 \%)$

$10(12.8 \%) \quad 6(10.9 \%)$

0.064

$\begin{array}{ll}74(94.9 \%) & 46(83.6 \%) \\ 4(5.13 \%) & 9(16.4 \%)\end{array}$




\section{Cureus}

Fatigue

0.894

No

59 (75.6\%)

$43(78.2 \%)$

Yes

$19(24.4 \%)$

$12(21.8 \%)$

Altered consciousness

No

$70(89.7 \%) \quad 51(92.7 \%)$

Yes

$8(10.3 \%)$

$4(7.27 \%)$

Vitals on hospital arrival

Death $(\mathrm{N}=78)$

Discharged $(\mathrm{N}=55)$

$\mathrm{p}$-Value

$\mathrm{HR}^{*}$

$105(21.2)$

105 (22.6)

0.945

SBP*

$128(27.7)$

$136(24.2)$

0.131

DBP*

$71.8(14.4)$

$78.4(12.7)$

0.009

Sats on RA*

$84.1(12.7)$

$83.7(12.4)$

0.864

Temp*

$38.0(0.95)$

$37.9(1.03)$

0.884

$\mathrm{RR}^{*}$

$30.6(9.40)$

$29.8(10.1)$

0.732

Labs on Day 1 in ICU

Death $(\mathrm{N}=78)$

Discharged $(\mathrm{N}=55)$

p-Value

$\mathrm{Hb}^{*}$

$122(32.9)$

121 (29.1)

0.839

WBC*

$15.1(11.8)$

10.9 (5.35)

0.007

Lymphocyte count*

$1.16(1.50)$

$1.27(1.08)$

0.618

Platelet count ${ }^{\star}$

277 (157)

281 (135)

0.884

CRP*

162 (139)

322 (1228)

0.383

$\mathrm{PCT}^{*}$

$10.6(31.9)$

$8.06(24.2)$

0.617

D-Dimer

2485 (3761)

2621 (5694)

0.882

$\mathrm{LDH}^{*}$

Ferritin ${ }^{*}$

745 (348)

515 (209)

$<0.001$

Lactate*

1895 (3177)

2363 (8652)

0.726

$3.92(3.82)$

2.20 (1.77)

0.003

Creatinine $^{*}$

3578 (29771)

111 (171)

0.307

Urea*

$18.3(13.5)$

$11.7(10.0)$

0.002

ALT $^{*}$

132 (466)

52.4 (48.0)

0.139

AST $^{*}$

223 (715)

68.8 (72.6)

0.063

$29.1(6.48)$

$31.9(4.63)$

0.004

Albumin

589 (1727)

1637 (6817)

0.274

Ventilation

Death

Discharged

$p$-value

Arrival at ICU on

$54(69.2 \%)$

8 (14.5\%)

NIV

$24(30.8 \%)$

47 (85.5\%)

Eventually required MV

$<0.001$

No

$0(0.00 \%)$

$35(63.6 \%)$

Yes

$78(100 \%)$

20 (36.4\%)

Intubated on ICU day

2.50 (3.02)

$0.96(1.82)$

$<0.001$

Duration of MV (days) ${ }^{\star}$

$7.03(6.52)$

3.51 (6.59)

0.003

Tracheostomy 


\section{Cureus}

No

Yes

Extubated successfully

No

Was never intubated

Yes

Treatments

Antibiotics

No

Yes

Antiviral

No

Oseltamivir

Oseltamivir and Lopinavir/Ritonavir

Plasma exchange

No

Yes

Tocilizumab

No

Yes

Dexamethasone

No

Yes

Anakinra

No

Yes

IVIG

No

Yes

Nitric oxide

No

Yes

Proning

No

Yes

Complications

In-hospital complications

No

Yes

$\begin{array}{ll}75(96.2 \%) & 53(96.4 \%) \\ 3(3.85 \%) & 2(3.64 \%)\end{array}$

$74(94.9 \%) \quad 1(1.82 \%)$

$0(0.00 \%) \quad 35(63.6 \%)$

$4(5.13 \%) \quad 19(34.5 \%)$

Death $(\mathrm{N}=78)$

Discharged $(\mathrm{N}=55)$

$p$-value

0.081

$1(1.28 \%) \quad 5(9.09 \%)$

77 (98.7\%)

50 (90.9\%)

57 (73.1\%)

$43(78.2 \%)$

$16(20.5 \%)$

$10(18.2 \%)$

$5(6.41 \%)$

2 (3.64\%)

$62(79.5 \%)$

$51(92.7 \%)$

16 (20.5\%)

4 (7.27\%)

0.091

$\begin{array}{ll}63(80.8 \%) & 51(92.7 \%) \\ 15(19.2 \%) & 4(7.27 \%)\end{array}$

0.029

$\begin{array}{ll}46(59.0 \%) & 21(38.2 \%) \\ 32(41.0 \%) & 34(61.8 \%)\end{array}$

75 (96.2\%)

52 (94.5\%)

$3(3.85 \%)$

$3(5.45 \%)$

$76(97.4 \%)$

54 (98.2\%)

2 (2.56\%)

$1(1.82 \%)$

$<0.001$

$60(76.9 \%) \quad 55(100 \%)$

$18(23.1 \%) \quad 0(0.00 \%)$

0.112

$42(53.8 \%) \quad 38(69.1 \%)$

$36(46.2 \%) \quad 17(30.9 \%)$

Death $(\mathrm{N}=78)$

Discharged $(\mathrm{N}=55)$

$p-$ Value

$<0.001$ 


\section{Cureus}

In-hospital AKI

No

Yes

In-hospital cardiac arrest with ROSC

No

Yes

In-hospital coagulopathy

No

Yes

Types of coagulopathies

DIC

DIC and LL ischemia

DIC, LL ischemi, bowel ischemia and renal ischemia

DVT

LL ischemia

No

$\mathrm{PE}$

In-hospital MI

No

Yes

In-hospital CVA

No

Yes

In-hospital pneumothorax

No

Yes

In-hospital UGI bleed

No

Yes

In-hospital rhabdomyolysis

No

Yes

In-hospital DKA

No

Yes

In-hospital infective endocarditis

No

Yes

Shock

Cardiogenic shock
$30(38.5 \%)$

$42(76.4 \%)$

$48(61.5 \%)$

$13(23.6 \%)$

$68(87.2 \%)$

$55(100 \%)$

$10(12.8 \%)$

$0(0.00 \%)$

0.627

$66(84.6 \%) \quad 49(89.1 \%)$

$12(15.4 \%) \quad 6(10.9 \%)$

0.019

$9(11.5 \%) \quad 1(1.82 \%)$

$1(1.28 \%) \quad 1(1.82 \%)$

$1(1.28 \%) \quad 0(0.00 \%)$

$0(0.00 \%) \quad 1(1.82 \%)$

$1(1.28 \%) \quad 0(0.00 \%)$

$66(84.6 \%) \quad 49(89.1 \%)$

$0(0.00 \%) \quad 3(5.45 \%)$

$73(93.6 \%) \quad 52(94.5 \%)$

$5(6.41 \%) \quad 3(5.45 \%)$

0.027

$78(100 \%) \quad 51(92.7 \%)$

$0(0.00 \%) \quad 4(7.27 \%)$

$72(92.3 \%) \quad 53(96.4 \%)$

$6(7.69 \%) \quad 2(3.64 \%)$

0.122

$69(88.5 \%) \quad 53(96.4 \%)$

$9(11.5 \%) \quad 2(3.64 \%)$

0.447

$75(96.2 \%) \quad 51(92.7 \%)$

$3(3.85 \%) \quad 4(7.27 \%)$

1

$76(97.4 \%) \quad 53(96.4 \%)$

$2(2.56 \%) \quad 2(3.64 \%)$

1

$76(97.4 \%) \quad 53(96.4 \%)$

$2(2.56 \%) \quad 2(3.64 \%)$

$<0.001$

$5(6.41 \%) \quad 0(0.00 \%)$ 


\section{Cureus}

No

Septic shock
Scores
NEWS2*
MEWS*
qCSI $^{*}$
$4 C^{*}$
VACO* $^{*}$
MuIBTSA

$19(24.4 \%)$

54 (69.2\%)

Death $(\mathrm{N}=78)$

6.63 (2.82)

3.51 (2.12)

$3.93(2.60)$

11.5 (3.70)

$10.8(10.6)$

$9.90(2.62)$

10.7 (3.57)
$47(85.5 \%)$

$8(14.5 \%)$

Discharged ( $\mathrm{N}=55)$

$p$-Value

$5.88(2.56)$

0.148

$3.61(2.03)$

0.81

4.38 (2.36)

0.324

9.35 (3.55)

0.002

$5.80(7.67)$

0.002

9.22 (3.30)

0.207

9.10 (3.16)

0.012

\section{TABLE 9: Comparison of clinical characteristics and mortality}

*Mean (SD).

ICU: intensive care unit, ED: emergency department, ACEI: angiotensin-converting enzyme inhibitor, COPD: chronic obstructive pulmonary disease, CKD: chronic kidney disease, OSA: obstructive sleep apnea, HR: heart rate, SBP: systolic blood pressure, DBP: diastolic blood pressure, RA: room air, RR: diastolic blood pressure, Hb: hemoglobin, WBC: white blood count, CRP: C-reactive protein, PCT: procalcitonin, LDH: lactate dehydrogenase, ALT: alanine transaminase, AST: aspartate aminotransferase,MV: mechanical ventilator, IVIG: intravenous immunoglobulin, ROSC: return of spontaneous circulation, AKI: acute kidney injury, PE: pulmonary embolism, DIC: disseminated intravascular coagulation, LL: lower limb, DVT: deep vein thrombosis, MI: myocardial infarction, CVA: cerebrovascular accident, UGI: upper gastrointestinal, DKA: diabetic ketoacidosis, MEWS: Modified Early Warning Score for Clinical Deterioration, NEWS2: National Early Warning Score 2, qCSI: Quick COVID-19 Severity Index, 4C score: International Severe Acute Respiratory Infection Consortium Clinical Characterization Protocol (4C mortality score for COVID-19), VACO Index: Veterans Health Administration COVID-19 Index for COVID-19 Mortality, MuLBSTA: MuLBSTA Score for Viral Pneumonia Mortality, PRIEST score: Pandemic Respiratory Infection Emergency System Triage Severity Score.

Among the patients who arrived at the ICU on MV, more patients died (69.2\%) than were discharged (14.5\%; $\mathrm{p}<0.001$ ). Furthermore, among the patients who died, $69.2 \%$ had arrived on MV while those discharged most (85.5\%) had arrived at ICU on non-invasive ventilation (NIV). In the mortality group, all patients eventually required MV at some stage during their ICU stay $(\mathrm{p}<0.001)$. On average, the patients who died were intubated between day 2 and day 3 in ICU, while discharged patients were intubated on average within the first ICU day.

Most of the patients in both groups had not received antiviral therapy (Oseltamivir \pm Kaletra). Plasma exchange was performed for $20.5 \%$ of the patients who died, and for $7.27 \%$ of those discharged. Out of the 78 patients who died, 32 (41.1\%) received Dexamethasone. Anakinra was used only for $3.85 \%$ of the patients who died, and for $5.45 \%$ of the discharged patients. Intravenous immunoglobulin (IVIG) was used in a minority of patients who died (2.56\%), and less frequently among discharged patients (1.82\%). Among the patients who died, $46.2 \%$ needed proning, while among the discharged patients $30.9 \%$ required proning. Inhospital complications developed in most of the patients who died (94.9\%), compared to $54.5 \%$ of those who were discharged $(\mathrm{p}<0.001)$. The most prominent of which were AKI, cardiac arrest, coagulopathy, and septic shock.

The average MEWS score was similar in the two groups of patients, however, the average NEWS2 severity score, qCSI score, and MulBTSA score were all on average higher in patients who died than those who were discharged (Table 10). The 4C and VACO scores were on average higher for the patients who died than those for discharged patients $(\mathrm{p}=0.002)$. While the average PRIEST COVID-19 clinical severity score was also higher amongst those who died ( $\mathrm{p}$ 0.012). 


\section{Cureus}

\begin{tabular}{|l|l|}
\hline Scores & $\mathbf{N}=133^{7}$ \\
\hline NEWS2 & $6.00(5.00,8.00)$ \\
Unknown & 19 \\
MEWS & $3.00(2.00,5.00)$ \\
Unknown & 17 \\
qCSI & $5(2.00,7.00)$ \\
Unknown & 12 \\
4C & $11.0(8.0,13.0)$ \\
Unknown & 17 \\
VACO (\%) & $5(0,12)$ \\
MulBTSA & $9.00(7.00,12.00)$ \\
PRIEST COVID-19 clinical severity score & $10.0(8.0,12.0)$ \\
Unknown & 13 \\
\hline
\end{tabular}

\section{TABLE 10: Early warning scores}

$7 \mathrm{n}(\%)$; median (IQR).

MEWS: Modified Early Warning Score for Clinical Deterioration, NEWS2: National Early Warning Score 2, qCSI: Quick COVID-19 Severity Index, 4C score: International Severe Acute Respiratory Infection Consortium Clinical Characterization Protocol (4C mortality score for COVID-19), VACO Index: Veterans Health Administration COVID-19 Index for COVID-19 Mortality, MuLBSTA: MuLBSTA Score for Viral Pneumonia Mortality, PRIEST score: Pandemic Respiratory Infection Emergency System Triage Severity Score.

On average the COVID-19 scores were higher in the non-survivor group, as shown in Table 11 . The high values of sensitivity (true positive rate), specificity (true negative rate), and AUC metrics, calculated for each score, show that all scores have a contribution to the prediction of in-hospital COVID-19 mortality. The AUROC graphs for each score are displayed in Figure 1. 


\section{Cureus}

\begin{tabular}{|c|c|c|c|c|c|c|c|}
\hline $\begin{array}{l}\text { CoVID-19 } \\
\text { scores }\end{array}$ & $\begin{array}{l}\text { All cases }(n= \\
133)\end{array}$ & $\begin{array}{l}\text { Death } \\
(\mathrm{N}=78)\end{array}$ & $\begin{array}{l}\text { Discharged } \\
(\mathrm{N}=55)\end{array}$ & $\begin{array}{l}\text { Cut- } \\
\text { off }\end{array}$ & Sensitivity & Specificity & AUC (95\% Cl) \\
\hline NEWS2* & $6.35(2.74)$ & $6.63(2.82)$ & $5.88(2.56)$ & 7.5 & 0.7209 & 0.4085 & $\begin{array}{l}0.5780(0.4721- \\
0.6838)\end{array}$ \\
\hline MEWS ${ }^{x}$ & $3.55(2.07)$ & 3.51 (2.12) & $3.61(2.03)$ & 4.5 & 0.3913 & 0.7000 & $\begin{array}{l}0.5124(0.4051- \\
0.6197)\end{array}$ \\
\hline qCSI* & $4.12(2.50)$ & $3.93(2.60)$ & $4.38(2.36)$ & 3.5 & 0.7200 & 0.3944 & $\begin{array}{l}0.5423(0.4409- \\
0.6436)\end{array}$ \\
\hline $4 C^{*}$ & $10.67(3.78)$ & $11.5(3.70)$ & $9.35(3.55)$ & 9.5 & 0.5435 & 0.7286 & $\begin{array}{l}0.6682(0.5675- \\
0.7688)\end{array}$ \\
\hline VACO* & 8.72 (9.75) & $10.8(10.6)$ & $5.80(7.67)$ & 11.3 & 0.8545 & 0.4103 & $\begin{array}{l}0.6761(0.5837- \\
0.7686)\end{array}$ \\
\hline MulBTSA $^{*}$ & $9.62(2.93)$ & $9.90(2.62)$ & $9.22(3.30)$ & 14.5 & 0.0727 & 0.9872 & $\begin{array}{l}0.4214(0.3206- \\
0.5223)\end{array}$ \\
\hline PRIEST* & $10.03(3.49)$ & $10.7(3.57)$ & $9.10(3.16)$ & 11.5 & 0.8571 & 0.3944 & $\begin{array}{l}0.6452(0.5459- \\
0.7444)\end{array}$ \\
\hline
\end{tabular}

\section{TABLE 11: Early warning scores}

*Mean (SD).

MEWS: Modified Early Warning Score for Clinical Deterioration, NEWS2: National Early Warning Score 2, qCSI: Quick COVID-19 Severity Index, 4C score: International Severe Acute Respiratory Infection Consortium Clinical Characterization Protocol (4C mortality score for COVID-19), VACO Index: Veterans Health Administration COVID-19 Index for COVID-19 Mortality, MuLBSTA: MuLBSTA Score for Viral Pneumonia Mortality, PRIEST score: Pandemic Respiratory Infection Emergency System Triage Severity Score.

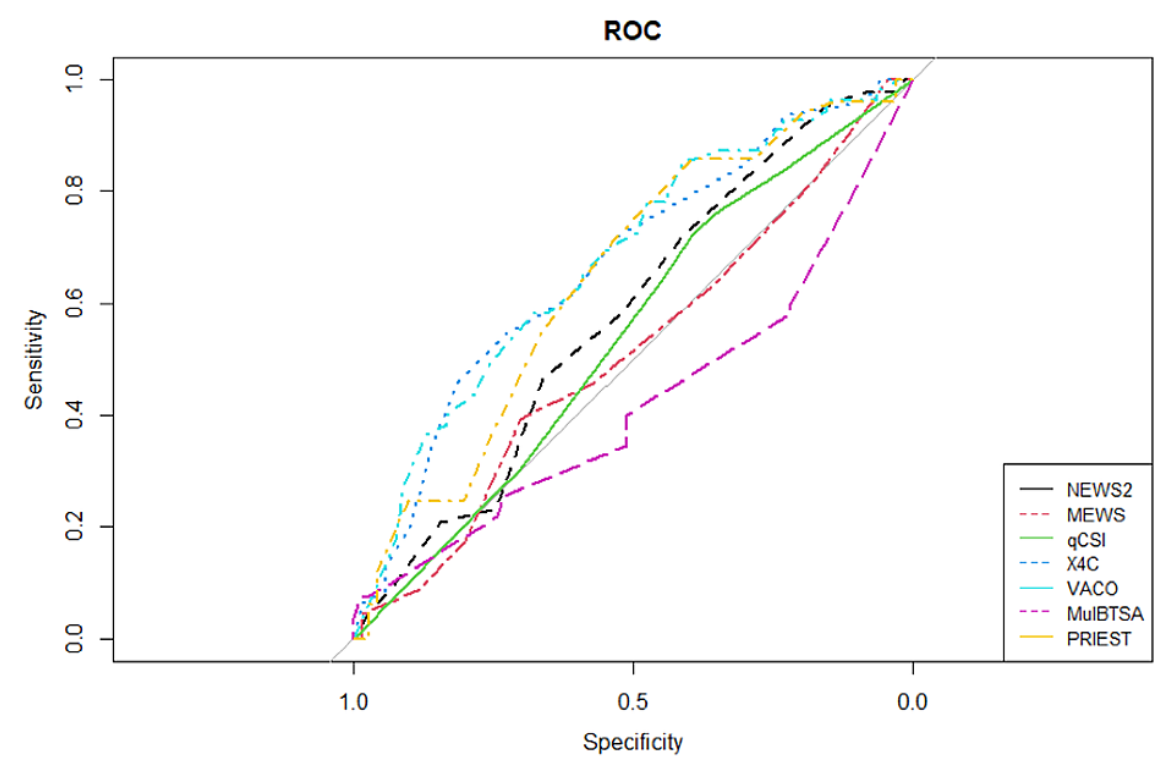

FIGURE 1: Area under the receiver operating characteristics graphs for each score

Table 12 shows statistically significant associations between in-hospital mortality from COVID-19 and age $(\mathrm{OR}=0.95)$, direct admission to ICU from ED ( $\mathrm{OR}=2.57)$, taking ACEI $(\mathrm{OR}=2.57)$, having $\mathrm{CKD}(\mathrm{OR}=0.25)$, WBC $(\mathrm{OR}=0.94), \mathrm{LDH}(\mathrm{OR}=1.00)$, lactate $(\mathrm{OR}=0.79)$, urea $(\mathrm{OR}=0.94)$, albumin $(0.004)(\mathrm{OR}=1.10)$, arrival to $\mathrm{ICU}$ on $\mathrm{MV}(\mathrm{OR}=12.8)$, delay in intubation $(\mathrm{OR}=0.69)$, duration of $\mathrm{MV}(\mathrm{OR}=0.91)$, Dexamethasone $(\mathrm{OR}=2.31)$, developing in-hospital complications ( $\mathrm{OR}=0.07$ ), in-hospital AKI ( $\mathrm{OR}=0.20)$, $4 \mathrm{C}$ mortality score for COVID19 (OR=0.85), VACO Index for COVID-19 mortality (OR=0.93), and PRIEST COVID-19 clinical severity score 


\section{Cureus}

$(\mathrm{OR}=0.87)$.

\begin{tabular}{|c|c|c|c|c|c|}
\hline & Death $(\mathrm{N}=78)$ & Discharged $(\mathrm{N}=55)$ & OR & p-Value (OR) & p-Value (overall) \\
\hline Age & $62.6(13.7)$ & $53.2(14.5)$ & $0.95[0.93 ; 0.98]$ & 0.001 & $<0.001$ \\
\hline Direct admission to ICU & & & & & 0.016 \\
\hline No (ward first) & $58(74.4 \%)$ & $29(52.7 \%)$ & Ref. & Ref. & \\
\hline Yes (from ED to ICU) & $20(25.6 \%)$ & $26(47.3 \%)$ & $2.57[1.24 ; 5.45]$ & 0.011 & \\
\hline Taking ACEI & & & & & 0.026 \\
\hline No & $63(80.8 \%)$ & $34(61.8 \%)$ & Ref. & Ref. & \\
\hline Yes & $15(19.2 \%)$ & $21(38.2 \%)$ & $2.57[1.18 ; 5.74]$ & 0.018 & \\
\hline CKD & & & & & 0.02 \\
\hline No & $59(75.6 \%)$ & $51(92.7 \%)$ & Ref. & Ref. & \\
\hline Yes & $19(24.4 \%)$ & 4 (7.27\%) & $0.25[0.07 ; 0.73]$ & 0.01 & \\
\hline DBP & $71.8(14.4)$ & $78.4(12.7)$ & $1.04[1.01 ; 1.07]$ & 0.015 & 0.009 \\
\hline WBC & $15.1(11.8)$ & $10.9(5.35)$ & $0.94[0.89 ; 0.99]$ & 0.017 & 0.007 \\
\hline LDH & 745 (348) & $515(209)$ & $1.00[1.00 ; 1.00]$ & $<0.001$ & $<0.001$ \\
\hline Lactate & $3.92(3.82)$ & $2.20(1.77)$ & $0.79[0.64 ; 0.96]$ & 0.02 & 0.003 \\
\hline Urea & $18.3(13.5)$ & $11.7(10.0)$ & $0.94[0.91 ; 0.98]$ & 0.005 & 0.002 \\
\hline Albumin & $29.1(6.48)$ & $31.9(4.63)$ & $1.10[1.03 ; 1.18]$ & 0.007 & 0.004 \\
\hline Arrival at ICU on & & & & & $<0.001$ \\
\hline MV & $54(69.2 \%)$ & $8(14.5 \%)$ & Ref. & Ref. & \\
\hline NIV & $24(30.8 \%)$ & 47 (85.5\%) & $12.8[5.45 ; 33.3]$ & $<0.001$ & \\
\hline Intubated on ICU day & $2.50(3.02)$ & $0.96(1.82)$ & $0.69[0.54 ; 0.90]$ & 0.005 & $<0.001$ \\
\hline Duration of mechanical ventilation (days) & $7.03(6.52)$ & $3.51(6.59)$ & $0.91[0.85 ; 0.97]$ & 0.005 & 0.003 \\
\hline Dexamethasone & & & & & 0.029 \\
\hline No & $46(59.0 \%)$ & $21(38.2 \%)$ & Ref. & Ref. & \\
\hline Yes & $32(41.0 \%)$ & $34(61.8 \%)$ & $2.31[1.14 ; 4.75]$ & 0.02 & \\
\hline In-hospital complications & & & & & $<0.001$ \\
\hline No & $4(5.13 \%)$ & $25(45.5 \%)$ & Ref. & Ref. & \\
\hline Yes & 74 (94.9\%) & 30 (54.5\%) & $0.07[0.02 ; 0.20]$ & $<0.001$ & \\
\hline In-hospital AKI & & & & & $<0.001$ \\
\hline No & $30(38.5 \%)$ & $42(76.4 \%)$ & Ref. & Ref. & \\
\hline Yes & $48(61.5 \%)$ & $13(23.6 \%)$ & $0.20[0.09 ; 0.42]$ & $<0.001$ & \\
\hline VACO & $10.8(10.6)$ & $5.80(7.67)$ & $0.93[0.89 ; 0.98]$ & 0.006 & 0.002 \\
\hline 4C & $11.5(3.70)$ & $9.35(3.55)$ & $0.85[0.76 ; 0.95]$ & 0.003 & 0.002 \\
\hline PRIEST COVID19 clinical severity score & $10.7(3.57)$ & $9.10(3.16)$ & $0.87[0.78 ; 0.98]$ & 0.018 & 0.012 \\
\hline
\end{tabular}

TABLE 12: Odds ratios of significant variables with $95 \%$ confidence interval and $p$-values

A multivariable logistic regression model was built, including the variables that showed statistically 
significant association with in-hospital mortality. Arrival to ICU on MV (OR=45.76), delay in intubation $(\mathrm{OR}=0.51)$, developing in-hospital complications $(\mathrm{OR}=0.1)$ and having $\mathrm{CKD}(0.21)$ remained significant, as shown in Table 13.

\begin{tabular}{|c|c|c|c|}
\hline Variable & p-Value & OR & C.I. (95\%) \\
\hline Arrival at ICU on MV & $<0.001$ & 45.76 & $(13.18,204.71)$ \\
\hline Day of intubation in ICU & 0.0006 & 0.51 & $(0.33,0.71)$ \\
\hline In-hospital complications & 0.006 & 0.1 & $(0.02,0.49)$ \\
\hline Having CKD & 0.07 & 0.21 & $(0.03,1.02)$ \\
\hline
\end{tabular}

TABLE 13: Multivariable analysis of clinical characteristics influencing COVID-19 mortality

\section{Discussion}

Based on our study of 133 patients admitted to the COVID-19 ICU, demographically the majority of the patients were older males, with underlying comorbidities, most commonly diabetes mellitus and hypertension. The majority developed in-hospital complications, namely AKI, cardiac arrest with the return of spontaneous circulation, and coagulopathy, and the rates of which were all higher in the mortality group. Risk factors that were statistically significant as determinants of in-hospital mortality were found to be older age, delayed ICU care, and previously having CKD. On the other hand, laboratory findings that were elevated in the mortality group were $\mathrm{LDH}$, lactate, urea, WBC, and albumin. It was observed that better outcomes were seen in those with early intubation during their ICU stay.

In our study, younger patients developed more favorable outcomes as compared to their older counterparts. The death rate was also noticed to be higher amongst males and those with co-morbidities. This is supported by a systematic review by Mehraeen et al. whereby older age and co-morbidities were found to be strong predictors of in-hospital admission and most importantly critical illness in patients with COVID-19 [20]. In a meta-analysis involving 18,012 patients and a systematic review of 15,794 patients, the most common comorbidities amongst critically ill COVID-19 patients was found to be diabetes and hypertension [21,22]. This paralleled with our results whereby those conditions predominated in our cohort.

However, there seems to be a debate on whether diabetes alone or synergistically with hypertension predicts severity and in-hospital mortality in those affected by the virus. Interestingly Sun et al. concluded that diabetes and raised blood glucose level, not a raised arterial blood pressure, is an independent predictor for the development of acute respiratory distress syndrome (ARDS) and subsequent respiratory failure in COVID-19 patients $[23,24]$. Nonetheless, the presence of co-morbidities in those affected by the virus equates to a higher likelihood of critical illness.

Extensive research has been undertaken to study the virulence of the SARS-CoV-2 virus, and it has been hypothesized that the virus gains entry into the host cells by binding to the angiotensin-converting enzyme 2 (ACE-2) receptors [24,25]. Such receptors are expressed by epithelial cells of the lungs, intestines, kidneys, brain, and blood vessels [24,25]. Animal studies have insinuated that ACEI and angiotensin-receptor blockers (ARBs) may increase the expression of ACE2 receptors [26]. This upregulation of the ACE2 receptor could potentially facilitate viral entry, providing an explanation for the increased severity of infection in those on ACEI or ARBs [24,25]. This can partly explain the high prevalence of diabetes and hypertension amongst critically ill COVID-19 patients since the majority of such patients are prescribed ACEI or ARBs $[27,28]$.

In contradiction to the aforementioned theory, a number of studies including ours, report findings of improved outcomes in patients being treated with the above medications. A multi-center study by Fang et al. revealed a lower risk of all-cause in-hospital mortality among hypertensive COVID-19 patients being treated with ACE-I or ARBs [28].

On the other hand, in Brazil, a randomized control trial (RCT) of 659 patients hospitalized with a mild to moderate COVID-19 infection who were on a prescription of ACEIs or ARBs prior to hospitalization, investigated the effect of continuing versus discontinuing ACEIs or ARBs during the hospital stay. No difference in outcome was observed when comparing both groups [29]. The lack of consistency in the current literature is apparent, nevertheless, it can be safe to say that there is no role in discontinuing ACEIs or ARBs among patients hospitalized with COVID-19 provided there is an indication for treatment.

In our cohort, the presence of CKD rendered the patients more susceptible to in-hospital mortality. It is well established that CKD is an independent risk factor for severe COVID-19 disease and mortality, after 
adjusting for other concurring comorbidities [30]. In a meta-analysis involving 1389 patients, those with a previous history of CKD are at a threefold increased risk of developing severe COVID-19 disease [31]. Furthermore, it is in the etiological nature of CKD that such patients will also suffer from multiple concomitant comorbidities. The uremia-induced immune dysfunction associated with CKD has been suggested to play a role in the severity of infections in such patients [32]. It is safe to say that the presence of CKD as a comorbidity is a significant poor prognostic factor in COVID-19 patients, in addition to being a reason for prioritizing these patients for vaccination.

The development of in-hospital complications was observed more commonly in those who died, with the most significant being AKI, cardiac arrest with ROSC, coagulopathy, and septic shock. These predominated amongst the deceased group as opposed to those discharged and were similarly witnessed in multiple studies $[33,34]$.

We observed other factors that increase the probability of in-hospital mortality, including delays in ICU admission and delays in intubation. Optimal timing for intubation and the definition of "early" intubation in patients with severe acute hypoxemic respiratory failure remains a controversial topic. It is no doubt that MV is associated with its own immediate and delayed complications, however, deferred intubation may also have detrimental consequences. Generally speaking in critically ill patients requiring intubation, it was previously established that a two-day delay in intubation is associated with increased mortality during the hospital stay [35].

This was supported more recently in a multi-center study conducted in New York specifically on COVID-19 patients. Similar to our findings, the study showed that in patients requiring MV for severe COVID-19, delays in intubation after admission was associated with higher mortality [36]. This is a crucial point to keep in mind during counseling patient's relatives and clinical decision-making.

On the other hand, Papousti et al. conducted a systematic review and a meta-analysis, whereby "early" intubation was defined as intubation within 24 hours from admission. Findings were reported showing no statistically detectable difference in all-cause mortality, duration of MV, and ICU length of stay between patients undergoing early versus late intubation [37].

Fundamentally, decisions on the timing of intubation should rely on the clinical judgment of experienced intensivists while keeping in mind the oxygen status and the level of respiratory distress the patient is experiencing. In those requiring higher oxygen support, our center prefers a trial of high flow nasal oxygen (HFNO) or NIV rather than proceeding directly to intubation, after risk/benefit evaluation and taking into consideration efficient resource allocation [38].

Steroid therapy has shown to be the sole intervention to significantly reduce mortality in patients with COVID-19. As reported by the RECOVERY TRIAL, dexamethasone specifically has proven its efficacy in reducing mortality when compared to conventional therapy [39]. It has been postulated that glucocorticoids down-regulate the pathogenesis of the virus and its subsequent complications by means of modulating the host inflammatory pathways, reducing vascular and endothelial wall inflammation, and in turn reducing organ and tissue injury, edema formation, and the risk of arterial and venous occlusion [40]. Similarly, it was noticed in our study that more patients amongst the survival group were prescribed dexamethasone than those in the mortality group.

Many prognostic and early warning scores have been utilized in COVID-19 patients. They each vary in the clinical parameters included and ease of applicability, however, the ultimate goal of such scoring systems is to predict patient prognosis and/or in-hospital mortality. This serves as an adjunct to ease clinical decisionmaking, triaging, resource allocation, and/or patient counseling. For each of our patients, we calculated seven early warning scores that have been previously used in COVID-19 patients, and we examined their performance in predicting in-hospital mortality in our patient cohort. To the best of our knowledge, our study is one of few to compare seven early warning scores in COVID-19 patients admitted to the ICU.

The scores studied were the MEWS for clinical deterioration [12], the NEWS2 score [13], qCSI [14], the International Severe Acute Respiratory Infection Consortium Clinical Characterization Protocol (4C mortality score for COVID-19) [15], the VACO index for COVID-19 mortality [16], the MuLBSTA Score for Viral Pneumonia Mortality (MulBTSA) [17], and the PRIEST [18]. All scores were observed to have the ability to predict in-hospital mortality, however, the 4C, VACO, and the PRIEST severity scores yielded significant findings compared to the remaining four scores. The NEWS, MEWS, qCSI scores mainly focused on vital sign stability, i.e., respiratory rate, saturation, temperature, pulse, and blood pressure. Whereas, the 4C, VACO, and PRIEST scores had a wider range of variables, especially the inclusion of comorbidities, gender, and age, the importance of which has been aforementioned and discussed as strong predictors of severity and mortality, which allowed for a more comprehensive assessment of each patient [41].

The 4C, VACO, and PRIEST scores proved to be the three most superior scores, having the highest AUC (area under the ROC Curve) values. The VACO and the PRIEST scores had the highest sensitivity of all the seven examined scores, while the sensitivity of the $4 \mathrm{C}$ score was satisfactory. However, the specificity of the $4 \mathrm{C}$ 
score was exceedingly higher than that of the VACO and PRIEST scores. When comparing the three most accurate scores, the $4 \mathrm{C}$ score proved to be less sensitive yet the most specific when compared to the VACO and PRIEST scores (Table 11).

\section{Limitations}

Our study is accompanied by a few limitations. First, the limited sample size, our study is a single-center experience and due to the lack of an electronic-based system linking our COVID-19 centers, a multi-center study was not undertaken. In addition, certain clinical parameters that could potentially influence the outcome of COVID-19 patients were not included, for example, chest X-ray findings, arterial blood gas analysis, patient weight/body mass index, and ventilator parameters. Also, due to the nature of the study, we could not assess the long-term complications and/or death rate in those who were discharged from the ICU.

\section{Conclusions}

The early identification of those likely to have an unfavorable prognosis allows for efficient triaging, adequate resource allocation, and the delivery of a high standard of care. In our experience certain demographic and clinical parameters were identified as early predictors of an adverse outcome; furthermore, the use of early warning scores can aid in clinical decision-making. In our experience, the 4C mortality score for COVID-19, the VACO index for COVID-19 mortality, and the PRIEST COVID-19 clinical severity score proved to be superior compared to the rest of the scores, leaving the choice down to personal preference and convenience in applicability.

\section{Additional Information \\ Disclosures}

Human subjects: Consent was obtained or waived by all participants in this study. Ethical Committee of the Ministry of Health, Kuwait issued approval NA. Animal subjects: All authors have confirmed that this study did not involve animal subjects or tissue. Conflicts of interest: In compliance with the ICMJE uniform disclosure form, all authors declare the following: Payment/services info: All authors have declared that no financial support was received from any organization for the submitted work. Financial relationships: All authors have declared that they have no financial relationships at present or within the previous three years with any organizations that might have an interest in the submitted work. Other relationships: All authors have declared that there are no other relationships or activities that could appear to have influenced the submitted work.

\section{Acknowledgements}

We would like to thank Dr. Meznah AlMutairi for her contribution to this research, by facilitating the logistics of ethical approval and data collection.

\section{References}

1. Coronavirus disease (COVID-19) dashboard . (2021). Accessed: July 12, 2021: https://covid19.who.int/.

2. Li Q, Guan X, Wu P, et al.: Early transmission dynamics in Wuhan, China, of novel Coronavirus-infected pneumonia. N Engl J Med. 2020, 382:1199-207. 10.1056/NEJMoa2001316

3. Coronavirus disease (COVID-19): How it is transmitted? . (2021). Accessed: July 12, 2021: https://www.who.int/news-room/q-a-detail/coronavirus-disease-covid-19-how-is-it-transmitted.

4. Novel Coronavirus (2019-nCoV) situation report-7 . (2021). Accessed: May 23, 2021: https://www.who.int/docs/default-source/coronaviruse/situation-reports/20200127-sitrep-7-2019-ncov.pdf.

5. Symptoms of novel Coronavirus (2019-nCoV) . (2021). Accessed: May 23, 2021: https://www.cdc.gov/coronavirus/2019-ncov/symptoms-testing/symptoms.html

6. Clinical spectrum of SARS-CoV-2 infection. (2021). Accessed: May 23, 2021: https://www.covid19treatmentguidelines.nih.gov/overview/clinical-spectrum.

7. Vincent JL, Taccone FS: Understanding pathways to death in patients with COVID-19. Lancet Respir Med. 2020, 8:430-2. 10.1016/S2213-2600(20)30165-X

8. Phua J, Weng L, Ling L, et al.: Intensive care management of coronavirus disease 2019 (COVID-19): challenges and recommendations. Lancet Respir Med. 2020, 8:506-17. 10.1016/S2213-2600(20)30161-2

9. Zangrillo A, Beretta L, Silvani P, et al.: Fast reshaping of intensive care unit facilities in a large metropolitan hospital in Milan, Italy: facing the COVID-19 pandemic emergency. Crit Care Resusc. 2020, 22:91-4.

10. Wu Z, McGoogan JM: Characteristics of and important lessons from the Coronavirus Disease 2019 (COVID19) outbreak in China: summary of a report of 72314 cases from the Chinese center for disease control and prevention. JAMA. 2020, 323:1239-42. 10.1001/jama.2020.2648

11. Wu C, Chen X, Cai Y, et al.: Risk factors associated with acute respiratory distress syndrome and death in patients with Coronavirus Disease 2019 Pneumonia in Wuhan, China. JAMA Intern Med. 2020, 180:934-43. 10.1001/jamainternmed.2020.0994

12. Subbe CP, Kruger M, Rutherford P, Gemmel L: Validation of a modified Early Warning Score in medical admissions. OJM. 2001, 94:521-6. 10.1093/qimed/94.10.521

13. Smith GB, Redfern OC, Pimentel MA, et al.: The National Early Warning Score 2 (NEWS2). Clin Med (Lond). 2019, 19:260. 10.7861/clinmedicine.19-3-260 
14. Haimovich AD, Ravindra NG, Stoytchev S, et al.: Development and validation of the quick covid-19 severity index: a prognostic tool for early clinical decompensation. Ann Emerg Med. 2020, 76:442-53. 10.1016/j.annemergmed.2020.07.022

15. Knight SR, Ho A, Pius R, et al.: Risk stratification of patients admitted to hospital with covid-19 using the ISARIC WHO Clinical Characterisation Protocol: development and validation of the 4C Mortality Score. BMJ. 2020, 370:m3339. 10.1136/bmj.m3339

16. King JT Jr, Yoon JS, Rentsch CT, et al.: Development and validation of a 30-day mortality index based on pre-existing medical administrative data from 13,323 COVID-19 patients: the Veterans Health Administration COVID-19 (VACO) Index. PLoS One. 2020, 15 :e0241825. 10.1371/journal.pone.0241825

17. Guo L, Wei D, Zhang X, Wu Y, Li Q, Zhou M, Qu J: Clinical features predicting mortality risk in patients with viral pneumonia: The MuLBSTA score. Front Microbiol. 2019, 10:2752. 10.3389/fmicb.2019.02752

18. Goodacre S, Thomas B, Sutton L, et al.: Derivation and validation of a clinical severity score for acutely ill adults with suspected COVID-19: the PRIEST observational cohort study. PLoS One. 2021, 16:e0245840. 10.1371/journal.pone.0245840

19. Kuwait confirms 3 coronavirus cases coming from Iran's Mashad . (2020). Accessed: July 12, 2021: https://www.kuna.net.kw/ArticleDetails.aspx?id=2864385 \&language=en.

20. Mehraeen E, Karimi A, Barzegary A, et al.: Predictors of mortality in patients with COVID-19: a systematic review. Eur J Integr Med. 2020, 40:101226. 10.1016/j.eujim.2020.101226

21. Wang B, Li R, Lu Z, Huang Y: Does comorbidity increase the risk of patients with COVID-19: evidence from meta-analysis. Aging (Albany NY). 2020, 12:6049-57. 10.18632/aging.103000

22. Barrera FJ, Shekhar S, Wurth R, et al.: Prevalence of diabetes and hypertension and their associated risks for poor outcomes in covid-19 patients. J Endocr Soc. 2020, 4:bvaa102. 10.1210/jendso/bvaa102

23. de Almeida-Pititto B, Dualib PM, Zajdenverg L, et al.: Severity and mortality of COVID 19 in patients with diabetes, hypertension and cardiovascular disease: a meta-analysis. Diabetol Metab Syndr. 2020, 12:75. 10.1186/s13098-020-00586-4

24. Sun Y, Guan X, Jia L, et al.: Independent and combined effects of hypertension and diabetes on clinical outcomes in patients with COVID-19: A retrospective cohort study of Huoshen Mountain Hospital and Guanggu Fangcang Shelter Hospital. J Clin Hypertens (Greenwich). 2021, 23:218-31. 10.1111/jch.14146

25. Wan Y, Shang J, Graham R, Baric RS, Li F: Receptor recognition by the novel Coronavirus from Wuhan: an analysis based on decade-long structural studies of SARS Coronavirus. J Virol. 2020, 94:e00127-20. 10.1128/JVI.00127-20

26. Vaduganathan M, Vardeny O, Michel T, McMurray JJ, Pfeffer MA, Solomon SD: Renin-angiotensinaldosterone system inhibitors in patients with Covid-19. N Engl J Med. 2020, 382:1653-9. 10.1056/NEJMsr2005760

27. Ferrario CM, Jessup J, Chappell MC, et al.: Effect of angiotensin-converting enzyme inhibition and angiotensin II receptor blockers on cardiac angiotensin-converting enzyme 2. Circulation. 2005, 111:260510. 10.1161/CIRCULATIONAHA.104.510461

28. Fang L, Karakiulakis G, Roth M: Are patients with hypertension and diabetes mellitus at increased risk for COVID-19 infection?. Lancet Respir Med. 2020, 8:e21. 10.1016/S2213-2600(20)30116-8

29. Chen AT, Coura-Filho GB, Rehder MH: Clinical characteristics of Covid-19 in China . N Engl J Med. 2020, 382:1860. 10.1056/NEJMc2005203

30. Lopes RD, Macedo AV, de Barros E Silva PG, et al.: Effect of discontinuing vs continuing angiotensinconverting enzyme inhibitors and angiotensin ii receptor blockers on days alive and out of the hospital in patients admitted with Covid-19. JAMA. 2021, 325:254-64. 10.1001/jama.2020.25864

31. Pakhchanian H, Raiker R, Mukherjee A, Khan A, Singh S, Chatterjee A: Outcomes of COVID-19 in CKD patients: a multicenter electronic medical record cohort study. Clin J Am Soc Nephrol. 2021, 16:785-6. 10.2215/CJN.13820820

32. Henry BM, Lippi G: Chronic kidney disease is associated with severe coronavirus disease 2019 (COVID-19) infection. Int Urol Nephrol. 2020, 52:1193-4. 10.1007/s11255-020-02451-9

33. Syed-Ahmed M, Narayanan M: Immune dysfunction and risk of infection in chronic kidney disease . Adv Chronic Kidney Dis. 2019, 26:8-15. 10.1053/j.ackd.2019.01.004

34. Shehab M, Alrashed F, Shuaibi S, Alajmi D, Barkun A: Gastroenterological and hepatic manifestations of patients with COVID-19, prevalence, mortality by country, and intensive care admission rate: systematic review and meta-analysis. BMJ Open Gastroenterol. 2021, 8: 10.1136/bmjgast-2020-000571

35. Puelles VG, Lütgehetmann M, Lindenmeyer MT, et al.: Multiorgan and renal tropism of SARS-COV-2 . N Engl J Med. 2020, 383:590-2. 10.1056/NEJMc2011400

36. Bauer PR, Gajic O, Nanchal R, et al.: Association between timing of intubation and outcome in critically ill patients: a secondary analysis of the ICON audit. J Crit Care. 2017, 42:1-5. 10.1016/j.jcrc.2017.06.010

37. Papoutsi E, Giannakoulis VG, Xourgia E, Routsi C, Kotanidou A, Siempos II: Effect of timing of intubation on clinical outcomes of critically ill patients with COVID-19: a systematic review and meta-analysis of nonrandomized cohort studies. Crit Care. 2021, 25:121. 10.1186/s13054-021-03540-6

38. Schünemann HJ, Khabsa J, Solo K, et al.: Ventilation techniques and risk for transmission of Coronavirus disease, including COVID-19: a living systematic review of multiple streams of evidence. Ann Intern Med. 2020, 173:204-16. 10.7326/M20-2306

39. Horby P, Lim WS, Emberson JR, et al.: Dexamethasone in hospitalized patients with Covid-19 . N Engl J Med. 2021, 384:693-704. 10.1056/NEJMoa2021436

40. Ferrara F, Vitiello A: Efficacy of synthetic glucocorticoids in COVID-19 endothelites . Naunyn Schmiedebergs Arch Pharmacol. 2021, 394:1003-7. 10.1007/s00210-021-02049-7

41. Covino M, Sandroni C, Santoro M, et al.: Predicting intensive care unit admission and death for COVID-19 patients in the emergency department using early warning scores. Resuscitation. 2020, 156:84-91. 10.1016/j.resuscitation.2020.08.124 